\title{
Neutrino Properties Probed by Lepton Number Violating Processes
}

\author{
Sabin Stoica \\ Department of Fundamental Research, Horia Hulubei Foundation, P.O. Box MG-12, Bucharest-Magurele, Romania \\ Email: stoica.at.theory.nipne.ro, sabin.stoica.at.unescochair-hhf.r
}

Received July, 2013

\begin{abstract}
Study of neutrino properties is nowadays one of the most active domains of research in physics. On the one hand, fundamental properties of the neutrinos like their absolute mass, their character (are they Dirac or Majorana particles?) and the number of neutrino flavors, are still unknown. On the other hand, the knowledge of these properties are of great importance since the neutrinos are very abundant in nature and play a key role in nuclear and particle physics, astrophysics and cosmology. In addition, the results of the neutrino oscillation experiments have convincingly showed that neutrinos have mass and mix, in contradiction to the initial assumptions of the Standard Model. In this context there is an increased interest in the study of the Lepton Number Violating (LNV) processes, since they are capable to decide on the above mentioned neutrino properties. Since recently, the neutrinoless double beta $(0 v \beta \beta)$ decay was considered the only process able to distinguish between Dirac or Majorana neutrinos and to give a hint on the absolute mass of the electron neutrino. At present, the increased luminosity of the LHC experiments at CERN makes it feasable the search for LNV processes at LHC as well. Besides the neutrino character, these studies can also shed light on the existence of other types of neutrinos (the sterile neutrinos), than the three known ones. In this paper, I make a brief review on our present knowledge about the neutrino properties and on the way they can be probed by LNV processes at low- and high-energies. Particularly, I refer to the $0 v \beta \beta$ decay process and to the first attempts of searching of LNV processes in hadron collider experiments, particularly in LHC experiments at CERN-Geneva.
\end{abstract}

Keywords: Neutrinos; Neutrino Oscillations; Double Beta Decay; Neutrino Mass; Lepton Number Violation

\section{Introduction}

Results of the neutrino oscillation experiments have convincingly showed that neutrinos have mass and can oscillate from one flavor to another. They extend our understanding on the Standard Model (SM) and strongly encourage us for searching of beyond SM physics.

However, fundamental properties of the neutrinos as their absolute mass, their character (are they non-identical (Dirac particles) or identical (Majorana particles) with their anti-particles?), the number of neutrino flavors, the mechanism of their mass generation and their mass hierarchy, are still unknown. The knowledge of these properties is of fundamental importance for understanding the formation, composition and evolution of the universe, as well as for all the processes in which the neutrinos take part.

In this context there is a great interest for the study of LNV processes because they are capable to provide information on these unknown neutrino properties.

Since recently, the neutrinoless double beta decay $(0 v \beta \beta)$ was considered the only process capable to distinguish between Dirac or Majorana neutrinos and to give a hint on the absolute mass scale of the electron neutrino. At present, the increased luminosity of the LHC experiments at CERN makes it feasable the searching of LNV processes at LHC experiments, as well. They can bring complementary information to that which can be extracted from low-energy neutrino studies.

In this paper, I make a brief review on our present knowledge about the neutrino properties and on the way they can be probed by LNV processes at low and high energies. Particularly, I refer to the $0 v \beta \beta$ decay process and to the first attempts of searching of LNV processes in hadron collider experiments, particularly in LHC experiments at CERN-Geneva.

The paper is organized as follows: in the Section 2, I shortly present the actual status of our knowledge about the neutrino properties. In the section 3 , I refer to the $0 v \beta \beta$ process and to how one can extract the relevant neutrino parameters from the information provided by the study of this decay mode in connection with neutrino oscillation experiments. Section 4 is dedicated to the presentation of the LNV processes at high energy, and to the first attempts for their searching at hadron colliders, 
particularly at LHC experiments at CERN. The paper ends up with Section Conclusions, where I sum up the importance of the study of these LNV processes and I sketch some future prospects.

\section{Neutrino Properties}

From neutrino oscillation experiments we know that neutrinos have a non-zero mass and they can oscillate from one flavor to another, in contradiction with the initial assumtions of the SM. The squared mass differences between mass eigenstates and the mixing angles $\left(\theta_{12}, \theta_{23}\right.$, $\theta_{13}$ ) are measured within a few percentage accuracy by various experiments running in both terestrial and underground laboratories, and measuring neutrinos produced in Sun, Earth atmosphere, accelerators and reactors [1-8]. The actual values of these parameters are: 1) $\Delta \mathrm{m}^{2}{ }_{12}=$ $\Delta \mathrm{m}_{\text {sol }}^{2} \sim 7.58 \times 10^{-5} \mathrm{eV}^{2} ; \tan ^{2} \theta_{12} \sim 0.484 \rightarrow \theta_{12} \sim 35^{\circ}-$ measured in solar neutrino (underground) + KamLAND (reactor) experiments; 2) $\left|\Delta \mathrm{m}^{2}{ }_{13}\right|=\left|\Delta \mathrm{m}^{2}{ }_{32}\right|=\Delta \mathrm{m}_{\text {atm }}^{2} \sim$ $2.40 \times 10^{-3} \mathrm{eV}^{2} ; \sin ^{2} 2 \theta_{23} \sim 1.02 \rightarrow \theta_{23} \sim 45^{0}-$ measured in atmospheric (underground) $+\mathrm{K} 2 \mathrm{~K}$ (reactor) + MINOS (accelerator) experiments; 3) $\sin ^{2} 2 \theta_{13} \sim 0.092 ; \theta_{13} \sim 9^{0}$ Daya Bay (reactor) experiment. This last measurement (with 5.2 $\sigma$ statistical significance) allows an unambigous differentiation between oscillation 1-3 and the anomalous disappearance of anti-electron neutrinos from the reactor $v$ flux at RENO and Double-Chooz experiments, strengh tening the argument for existence of a sterile neutrino. Future expected measurements at T2K, RENO, MINOS, etc. Experiments are expected to start in the next future to measure the imbalance matter-antimatter. An important remark is the following: while the sign of $\Delta \mathrm{m}^{2}{ }_{12}$ can be measured due to matter effects of the neutrino propagation from sun, the sign of $\Delta \mathrm{m}^{2}{ }_{13}$ can not be measured. In a three flavor neutrino analysis this leads to two possible scenarios for the neutrino mass hierarchy, the socalled "normal" $\left(\mathrm{m}_{1}<\mathrm{m}_{2}<<\mathrm{m}_{3}\right)$ and "inverted" $\left(\mathrm{m}_{3}<<\mathrm{m}_{1}<\right.$ $\mathrm{m}_{2}$ ) hierarchies, which are shown in Figure 1. As we mentioned above, there remain still unknown issues as: the absolute mass of the neutrinos, the mechanism of mass generation, their mass hierarchy, if neutrinos are Majorana or Dirac particles?), are there "sterile" neutrinos, is the CP symmetry violated in the lepton sector, and if yes, how much? Information on all these issues can be provided by the study of LNV processes.

\section{Lepton Number Violating Processes at Low-Energy}

Lepton number (LN) conservation is a symmetry that is experimentally verified to a very high precision, and it is assumed within the SM. However, it is not a consequence of a known gauge symmetry, thus other theories more general than the SM allow the non-conservation of this quantum number. Lepton number violation was first invoked to argue the possible existence of the $0 v \beta \beta$ decay mode, a process which could probe the Dirac or Majorana character of neutrinos. More detailed information about its study can be found in some recent publications [9-12]. The Double beta decay (DBD) is a nuclear natural decay by which an even-even nucleus transforms an even-even nucleus into another even-even nucleus with the same mass but the nuclear charge changed by two units. It occurs whatever single $\beta$ decay can not occur due to energetical reasons, or it is highly forbidden by angular momentum selection rules. Figure 2 illustrates such a situation. Within the SM this process occurs with the emission of two electrons and two anti-neutrinos, and this decay mode $(2 v \beta \beta)$ was already measured for eleven isotopes. However, within theories that go beyond SM (BSM), in which LNV is permitted, the DBD process may occur without emission of neutrinos. This implies that neutrino is a Majorana particle with mass different from zero. Considering the most common mechanism of occurrence, i.e. exchange of light Majorana neutrinos between two nucleons inside the nucleus and in the presence of only left-handed (LH) weak interactions, the half-life of this decay mode can be expressed as a product of three factors:

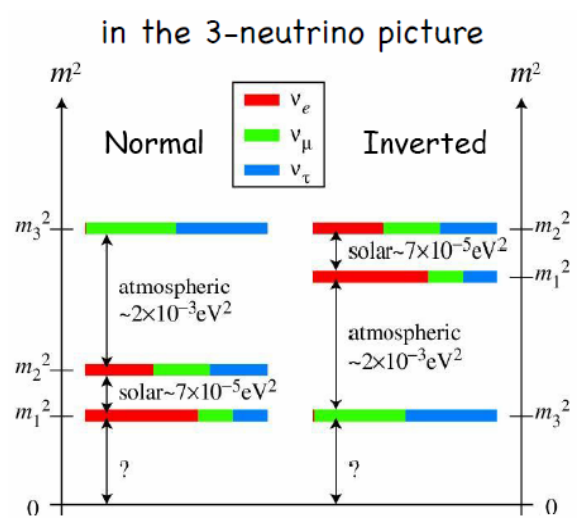

Figure 1. Hierarchical neutrino mass scheme: (a) normal hierarchy; (b) inverted hierarchy.

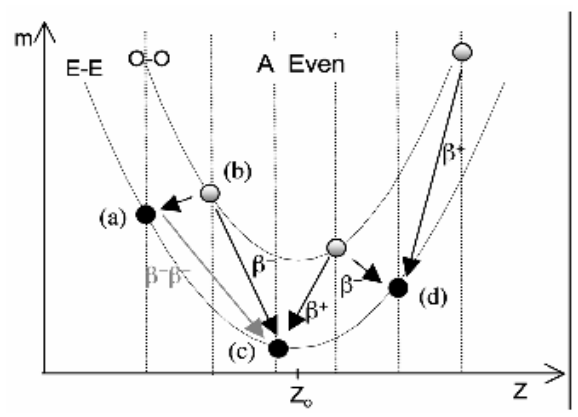

Figure 2. Illustration of a DBD process: nuclei (a) and (d) are stable against $\beta$ decay, but unstable against $\beta \beta$ decay: $\beta^{-} \beta^{-}$for (a) and $\beta^{+} \beta^{+}$for (d). 


$$
\left[\mathrm{T}^{0 v}{ }_{1 / 2}\right]^{-1}=\mathrm{G}^{0 v}\left(\mathrm{Q}_{\beta \beta}, \mathrm{Z}\right)\left|\mathrm{M}^{0 v}\right|^{2} \quad<\mathrm{m}_{v}{ }^{2}>/ \mathrm{m}_{\mathrm{e}}
$$

where $G^{0 v}$ is a phase space depending on the energy $Q_{\beta \beta}$ released in the decay and on the nuclear charge $Z$, and $\mathrm{M}^{0 v}$ are the nuclear matrix elements (NMEs) which depend on the nuclear structure of the isotope that decays. The third factor $\left\langle\mathrm{m}_{v}\right\rangle$ appearing in Equation (1) is a BSM parameter, that can be expressed in terms of neutrino mass eigenstates and neutrino mixing matrix $\mathrm{U}$ :

$$
<\mathrm{m}_{\mathrm{v}}>^{2}=\left|\Sigma_{\mathrm{i}} \mathrm{U}_{\mathrm{ei}}{ }^{2} \mathrm{~m}_{\mathrm{i}}\right|^{2}=\left.\left.\left|\Sigma_{\mathrm{i}}\right| \mathrm{U}_{\mathrm{ei}}\right|^{2} \mathrm{e}^{\mathrm{i} \alpha} \mathrm{m}_{\mathrm{i}}\right|^{2}, \mathrm{i}=1,2,3
$$

Experimentally one can distinguish the two DBD modes by measuring the sum of the electron energies: for the $2 v \beta \beta$ mode the number of DBD events versus the sum of the electron energies is a function that looks like a gaussian (the electrons share their energy with the two neutrinos), while in the case of $0 v \beta \beta$ mode this function is a single line at the energy $Q_{\beta \beta}$ (all the energy released in the decay is taken by the two electrons).

The extraction of the neutrino parameters is a very important issue. As one can see from the Equations (1) and (2) it is subject of the measured half-lives and of the precise calculation of the NMEs. Their accurate calculation is a challenge for the study of $0 v \beta \beta$. A measurement of the $0 v \beta \beta$ decay rate combined with neutrino oscillation data and a reliable calculation of the NMEs, would yield insight into all three neutrino mass eigenstates. In the following we show how the neutrino mass parameter can be extracted in the case that one (dominant) mechanism is responsible for the occurrence of $0 v \beta \beta$ decay. This is done separately for the two mass hierarchy scenarios.

$$
\begin{aligned}
& \text { i) normal hierarchy: } \\
& \begin{aligned}
\left|<m_{v}>\right| & =\mid \mathrm{c}_{13}^{2} \mathrm{~s}_{12}^{2}\left(\Delta \mathrm{m}_{\text {sun }}^{2}\right)^{1 / 2}+\mathrm{s}_{13}^{2}\left(\Delta \mathrm{m}_{\text {atm }}^{2}\right)^{1 / 2} \mathrm{e}^{2 \mathrm{i \alpha}} \\
& \leq 4 \cdot 10^{-3} \mathrm{eV}
\end{aligned}
\end{aligned}
$$

\section{ii) inverted hierarchy:}

$$
\begin{aligned}
& \left|<m_{v}>\right|=\mid \mathrm{c}_{13}^{2} \mathrm{~s}_{12}^{2}\left(\Delta \mathrm{m}_{\text {sun }}^{2}\right)^{1 / 2}+\left(\Delta \mathrm{m}_{\text {atm }}^{2}\right)^{1 / 2} \mathrm{~s}^{2}{ }_{13}\left(1-\sin ^{2} 2 \theta_{12}\right. \\
& \left.\sin \alpha_{12}\right)^{1 / 2}\left|\rightarrow 1.5 \cdot 10^{-2} \mathrm{eV} \leq\right|<m_{v}>\mid \leq 5.0 \cdot 10^{-2} \mathrm{eV} \text { (4) }
\end{aligned}
$$

where $\mathrm{c}_{\mathrm{ij}}=\cos \theta_{\mathrm{ij}} \quad$ and $\mathrm{s}_{\mathrm{ij}}=\sin \theta_{\mathrm{ij}} \quad$ are the neutrino mixing parameters, $\theta_{\mathrm{ij}}$ being the mixing angle between neutrino species $i$ and $j$. It is worth to mention that while in the case of normal hierarchy there is no lower limit for the electron neutrino mass, so an exact cancellation between the terms in Equation (3) can occur. By contrary, in the case of inverted hierarchy there is a lower limit for the electron neutrino mass and this will be checked by the next generation of the DBD experiments. This is an important experimental challenge in the study of this process. Theoretically, the main challenge is the accurate calculation of the NMEs involved in DBD. Then, if the $0 v \beta \beta$ will be discovered, an important issue will be to establish the (dominant) mechanism of its occurrence. In case when more mechanisms contribute to the $0 v \beta \beta$ occurrence, the expression of the half-life is modified accordingly. An interesting case is that when two mecha- nisms dominate: exchange of light (active) and heavy (sterile) Majorana neutrinos. Recently, it has shown that in spite of the naive expectation that the light neutrinos give the dominant contribution, heavy sterile neutrinos can saturate the present experimental bound of $0 v \beta \beta$ decay process. In this case, under the assumtion that only one flavour $(\mathrm{N})$ of heavy neutrino exist, the expression of the half-life reads:

$$
\begin{aligned}
& \left(\mathrm{T}^{0 v}{ }_{1 / 2}{ }^{-1}=\mathrm{G}^{0 v}\left(\mathrm{M}_{v} \eta_{v}+\mathrm{M}_{\mathrm{N}} \eta_{\mathrm{N}}\right)^{2},\right. \text { with } \\
& \eta_{v}=<\mathrm{m}_{v}>_{3} / \mathrm{m}_{\mathrm{e}}=\Sigma_{\mathrm{i}} \mathrm{U}_{\mathrm{ei}}{ }^{2} \mathrm{~m}_{\mathrm{i}} / \mathrm{m}_{\mathrm{e}} \text { and } \\
& \eta_{\mathrm{N}}=<\mathrm{m}_{\mathrm{v}}>{ }_{4} / \mathrm{M}_{\mathrm{N}}=\mathrm{U}_{\mathrm{eN}}{ }^{2} \mathrm{~m}_{\mathrm{p}} / \mathrm{M}_{\mathrm{N}}
\end{aligned}
$$

and

$$
\begin{aligned}
<\mathrm{m}_{v}>_{3+1} & =\mid \mathrm{c}^{2}{ }_{12} \mathrm{c}^{2}{ }_{13} \mathrm{c}^{2}{ }_{14} \mathrm{e}^{2 \mathrm{i \alpha} \alpha} \mathrm{m}_{1}+\mathrm{c}^{2}{ }_{13} \mathrm{c}^{2}{ }_{14} \mathrm{~s}^{2}{ }_{12} \mathrm{e}^{2 \mathrm{i} \alpha 2} \mathrm{~m}_{2} \\
& +\mathrm{s}_{13}^{2} \mathrm{c}_{14} \mathrm{e}^{2 \mathrm{i \alpha} 3} \mathrm{~m}_{3}+\mathrm{s}^{2}{ }_{14} \mathrm{M}_{\mathrm{N}}
\end{aligned}
$$

In this scenario one can extract information about the neutrino parameters if we have data from $0 v \beta \beta$ decay of at least two different nuclei.

\section{Lepton Number Niolating Processes at High-Energies}

In the extensions of the SM where a Majorana mass term is introduced, the LN is violated by two units. Thus, any such a LNV process can probe the Majorana character of the neutrino. At present the increase of the integrated luminosity at the LHC and superB experiments makes feasible the study of LNV processes at high energy, as well. There are many possible decay channels where the $\mathrm{LN}$ is violated by two units. The common experimental signature of these channels is the presence of same sign di-leptons in the decay products. Below, we give a list of such possible decay channels. There are baryon, meson, tau, top quark, double Higgs possible decays, with 3-body or 4-body products in the final states.

i) baryon decays: $\mathrm{B} \rightarrow \mathrm{B}_{1}{ }^{ \pm} l_{2}^{ \pm}$

ii) hyperon decays: $\quad \Sigma^{-} \rightarrow \Sigma^{+} \mathrm{e}^{-} \mathrm{e}^{-} ; \Xi^{-} \rightarrow \mathrm{p} \mu^{-} \mu$ $\Xi_{c}^{+} \rightarrow \Xi^{-} \mu^{+} \mu^{+}$, etc.

iii) meson decays: $\mathrm{M}^{ \pm} \rightarrow \mathrm{M}^{-/+} 1_{1}^{ \pm} 1_{2}^{ \pm}$(3-body decays) $\mathrm{M}^{0} \rightarrow \mathrm{l}_{1}{ }^{-} \mathrm{l}_{2}^{-} \mathrm{M}_{1}^{+} \mathrm{M}_{2}^{+}$(4-body decays)

iv) tau decays:

$$
\tau^{-} \rightarrow \mu^{+} \mu^{-} \mu^{-} ; \tau-\rightarrow p \mu^{-} \mu^{-} \tau^{-} \rightarrow v_{\tau} 1^{-} 1^{-} X^{+}
$$

v) same sign di-leptonic production: $\mathrm{pp} \rightarrow \mathrm{1}_{1}{ }^{+} \mathrm{l}_{2}{ }^{+} \mathrm{X}$

vi) top-quark decay: $\mathrm{t} \rightarrow \mathrm{b}{1_{1}}^{+} \mathrm{l}_{2}^{+} \mathrm{W}^{-} \mathrm{W}^{-}$

vii) double-charged Higgs decays: $\mathrm{H}^{ \pm} \rightarrow \mathrm{l}_{1}^{ \pm} \mathrm{l}_{2}{ }^{ \pm} \mathrm{X}$

The decay sensitivity of different heavy flavor LNV processes is determined by comparing the scale of the neutrino mass with the energies of the decay process [13]. We have three different cases: 1) exchange of light neutrinos $\left(\mathrm{m}_{v}{ }^{2}<<\mathrm{q}^{2}\right)$. In this case the decay rate $\mathrm{R} \sim<\mathrm{m}_{\|}{ }>$ $=\Sigma_{\mathrm{i}} \mathrm{U}_{\mathrm{li}} \mathrm{U}_{\mathrm{li}^{\mathrm{i}}} \mathrm{m}_{\mathrm{i}}\left(\mathrm{U}_{\mathrm{li}}-\right.$ mixing parameters of the active neutrinos); 2) exchange of heavy neutrinos $\left(\mathrm{m}_{v}{ }^{2}>>\mathrm{q}^{2}\right)$, and in this case the decay rate $\rightarrow \mathrm{R} \sim<\mathrm{m}_{\mathrm{ll}}>=\Sigma_{\mathrm{N}} \mathrm{V}_{\mathrm{IN}} \mathrm{V}_{\mathrm{l}}$ 'N 
/ $\mathrm{m}_{\mathrm{N}}\left(\mathrm{V}_{\mathrm{IN}}-\right.$ mixing parameters between light (active) and heavy (sterile) neutrinos); and 3) exchange of neutrinos with mass of the of the order of the energy decay process $\left(\mathrm{m}_{\mathrm{N}}^{2} \sim \mathrm{q}^{2}\right)$. In this case they can be produced on their mass shell and the decay rates are enhanced due to the resonant effect associated to their decay widths $\Gamma_{\mathrm{N}} \rightarrow \mathrm{R}$ $\sim \Sigma_{\mathrm{N}} \mathrm{V}_{\mathrm{IN}} \mathrm{V}_{\mathrm{l}, \mathrm{N}} / \Gamma_{\mathrm{N}}$. For the first two cases the decay rates are quite small: in the first case due to the smallness of both the mixing parameters between the active neutrinos and of the neutrino mass (of $\sim 1 \mathrm{eV}$ ), and in the second case due to the smallness of the mixing parameters between the ligh (active) and heavy (sterile) neutrinos divided by the large mass of the heavy neutrino. The branching ratios $(\mathrm{Br})$ in these cases are of the order of $10^{-20}-10^{-31}$ and, at the present LHC luminosities, they can not be detected. The third case is quite interesting since the theoretical predictions for the $\mathrm{Br}$ for these resonant decay processes become at reach of the present and next future high energy experiments.

Until now several LNV processes at high energy have already been investigated. Their non-observation has set bounds on the corresponding $\mathrm{Br}$ and, further, on the neutrino mixing parameters. In the following we shortly present the most important such investigations performed at LHC experiments at CERN.

ATLAS experiment [14] has performed an inclusive search of events with two isolated leptons (e or $\mu$ ) having the same electric charge. The data are selected from events collected from pp collisions at $\operatorname{sqrt}(\mathrm{s})=7 \mathrm{TeV}$ by the ATLAS detector and correspond to an integrated luminosity of $34 \mathrm{pb}^{-1}$. The spectra in dilepton invariant mass are compared to SM predictions. No evidence is found for contributions beyond those of the SM. Limits are set on the cross-section in a fiducial region for new sources of same-sign high-mass dilepton events in the ee, $\mathrm{e} \mu$ and $\mu \mu$ channels. Four models predicting same-sign dilepton signals are constrained: two descriptions of Majorana neutrinos, a cascade topology similar to supersymmetry or universal extra dimensions, and fourth generation down-type quarks. Assuming a new physics scale of $1 \mathrm{TeV}$, Majorana neutrinos produced by an effective operator $\mathrm{V}$ with masses below $460 \mathrm{GeV}$ are excluded at $95 \% \mathrm{CL}$. A lower limit of $290 \mathrm{GeV}$ is set at $95 \% \mathrm{CL}$ on the mass of fourth generation down type quarks.

CMS experiment [15] has searched for events with same-sign isolated dileptons (ee, $\mathrm{e} \mu, \mu \mu, \mu \tau, \tau \tau$ ). The searches used an integrated luminosity of $35 \mathrm{pb}^{-1}$ of $\mathrm{pp}$ collision data at a ECM of $7 \mathrm{TeV}$ collected by the CMS experiment at the LHC. The observed numbers of events agree with the SM predictions, and no evidence for new physics was found. To facilitate the interpretation of the data in a broader range of new physics scenarios, information on the event selection, detector response, and efficiencies is provided.
LHCb experiment has investigated several LNV processes of meson and tau decays. A first search of same sign dileptons was performed in the decays of $\mathrm{B}^{+} \rightarrow \mathrm{K}^{-}$ $\left(\pi^{-}\right) \mu^{+} \mu^{+}$at an integrated luminosity of $36 \mathrm{pb}^{-1}[16]$. No signal was observed in either channels. They set limits of the $\mathrm{Br}$ for the two channels as follows: $\mathrm{Br}\left(\mathrm{B}^{+} \rightarrow \mathrm{K}^{-} \mu^{+} \mu^{+}\right)$ $<5.4 \cdot 10^{-8}$ and $\operatorname{Br}\left(\mathrm{B}^{+} \rightarrow \pi^{-} \mu^{+} \mu^{+}\right)<5.8 \cdot 10^{-8}$ at $95 \% \mathrm{CL}$, which improves the previous existed limits by factors of 40, 30, respectively. Another analysis was performed for the $\mathrm{B}^{-}$decays into same sign di-muon channels at an integrated luminosity of $380 \mathrm{pb}^{-1}$ [17]. Also, no signal was observed beyond the $\mathrm{SM}$ and limits were set for the channels $\mathrm{Br}\left(\mathrm{B}^{-} \rightarrow \mathrm{D}^{+} \mu^{-} \mu^{-}\right)<5.6 \cdot 10^{-7}$ and $\mathrm{B}\left(\mathrm{B}^{-} \rightarrow \mathrm{D}^{*^{+}} \mu^{-}\right.$ $\left.\mu^{-}\right)<4.1 \times 10^{-6}$ at $90 \% \mathrm{CL}$. Besides these B meson channels a first search for tau decays was also performed [18]. Particularly, such an investigated process was $\tau^{-} \rightarrow$ $\mu^{+} \mu^{-} \mu^{-}$, which is in the same time a Lepton Flavor Violating process. The analysis was done using $1.0 \mathrm{fb}^{-1}$ of data collected in 2011 at $(\mathrm{s})^{1 / 2}=7 \mathrm{TeV}$. The upper limit for the $\mathrm{Br}$ was $\mathrm{Br}<7.8 \cdot 10^{-8}$ at $95 \% \mathrm{CL}$. These studies performed at LHC experiments will be certainly improved in the next future with a new set of data at an increased luminosity. Besides these already investigated channels, there are many others that merit to be investigated, according to theoretical estimations for the Br. For example, the hyperon decay channels are at present weakly constraint $\left(\mathrm{Br} \sim 10^{-3}-10^{-4}\right)$. In addition, there are very recent theoretical estimations which show that 4-body decay channels, like decays of neutral mesons (B, D), or the tau decay channel $\tau^{-} \rightarrow v_{\tau} \mu^{-} \mu^{-} \pi^{+}$can provide us with even more stringent bounds on $\mathrm{Br}$ and neutrino mixing parameters between muon flavor and sterile flavor (N) [19]. Thus, the study of the LNV channels at high energies opens an interesting direction of investigation at LHC experiments and superB factories in the next future.

\section{Conclusions}

Recent neutrino oscillation experiments have convincingly shown that neutrinos are massive particles and they mix. This is the first evidence that extends our understanding on the SM and encourages us to search for BSM physics. The large majority of BSM theories involve massive Majorana neutrinos which imply non-conservation of the LN. Concerning the neutrino properties we still do not know important issues as the scale of their absolute mass and the mass hierarchy, the mechanism of their mass generation, the nature of neutrinos (are they Dirac or Majorana particles?), the number of neutrino flavors, etc. The LNV processes can shed light on these issues, that is why there is a great interest to search for such processes. At low energy there is the $0 v \beta \beta$ decay, a process which is intensively studied both theoretically and experimentally. Theoretically, a key challenge is to 
accurately compute the NMEs involved in DBD by developing of nuclear structure methods for their calculation. Experimentally, there are several running experiments, and others are planned to start in the next future. The expectation is to explore the entire region of neutrino masses associated with the inverted mass hierarchy scenario. If the $0 v \beta \beta$ will be discovered, the next challenge will be to find the dominant mechanism(s) which contributes to its occurrence. At present, a new opportunity appears: the search of LNV processes at high energies. The very large "effective luminosity" of $0 v \beta \beta$ decay experiments becomes now to be compensated by the increased luminosity at LHC and superB factories. Thus, the search of these processes at high energies becomes another interesting avenue of research. Also, the combined information from low- and high-energy experiments will be of help in the study of LNV processes with consequences on the better understanding of the neutrino properties.

\section{Acknowledgements}

This work was supported by a grant of the Romanian National Authority for Scientific Research, CNCS - UEFISCDI, project no. PN-II-ID-PCE-2011-3-0318.

\section{REFERENCES}

[1] Y. Ashie et al. (SK collaboration), "Measurement of Atmospheric Neutrino Oscillation Parameters by Super-Kamiokande I," Physical Review D, Vol. 71, No. 11, 2005. doi:10.1103/PhysRevD.71.112005

[2] T. Araki et al., "Measurement of Neutrino Oscillation with KamLAND: Evidence of Spectral Distortion," Physical Review Letters, Vol. 94, No. 8, 2005, 081801.doi:10.1103/PhysRevLett.94.081801

[3] M. H. Ahn et al., "Measurement of Neutrino Oscillation by the K2K Experiment," Physical Review D, Vol. 74, No. 7, 072003, 2006. doi:10.1103/PhysRevD.74.072003

[4] P. Adamson et al., (MINOS collaboration), "Measurement of the Neutrino Mass Splitting and Flavor Mixing by Minos," Physical Review Letters, Vol. 106, 181801,

\section{1. doi:10.1103/PhysRevLett.106.181801}

[5] Daya Bay Collaboration, Physical Review Letters, "Observation of Electron-Antineutrino Disappearance at Daya Bay," Vol. 108, 171803, 2012. doi:10.1103/PhysRevLett.108.171803

[6] J. Ahn et al., (RENO collaboration), "Observation of Reactor Electron Antineutrinos Disappearance in the RENO Experiment," Physical Review Letters, Vol. 108, 191802, 2012. doi:10.1103/PhysRevLett.108.191802

[7] Y. Abe et al., (Double Chooz collaboration), "Indication of Reactor $\bar{v}_{e}$ Disappearance in the Double Chooz Experimen," Physical Review Letters, Vol. 108, 131801, 2012. doi:10.1103/PhysRevLett.108.131801

[8] K. Abe et al., (T2K Collaboration), Physical Review D, Vol. 85, No. 3, 0311032012. doi:10.1103/PhysRevD.85.031103

[9] J. D. Vergados, H. Ejiri and F. Simkovic, arXiv: 1205.0649.

[10] A. Faessler, arXiv:1203. 3648.

[11] Werner Rodejohann, International Journal of Modern Physics E, Vol. 20, No. 09, 2011, 1833. doi:10.1142/S0218301311020186

[12] M. Horoi and S. Stoica. Physical Review C, Vol. 81, 024321, 2010; A. Neascu, S. Stoica and M. Horoi, Physical Review C, Vol. 86, 067304, 2012.

[13] A. Atre, T. Han, S. Pascoli and B. Zhang, JHEP, Vol. 05, 030 (2009).

[14] ATLAS collaboration, JHEP, Vol. 10, 107 (2011).

[15] CMS collaboration, JHEP, Vol. 06, 077 (2011).

[16] LHCb Collaboration, "Search for Lepton Number Violating Decays $\mathrm{B}+\rightarrow \pi-\mu+\mu+$ and $\mathrm{B}+\rightarrow \mathrm{K}-\mu+\mu+$," Physical Review Letters, Vol. 108, 101601, 2012. doi:10.1103/PhysRevLett.108.101601

[17] LHCb collaboration, Phys. Rev.85,112004 (2012).

[18] LHCb collaboration: J.Harrison, Nucl. Phys. B, Proceedings Supplement, 2012; LHCb collaboration: P. Seyfert, arXiv: 1209; 4939v1 [hep-ex] and FPCP 2012, Hefei, China.

[19] G. Lopez-Castro, N. Quinteros, arXiv: 1212.0037 [hep$\mathrm{ph}]$. 